\title{
ARTICLE
}

\section{Age-of-diagnosis dependent ileal immune intensification and reduced alpha-defensin in older versus younger pediatric Crohn Disease patients despite already established dysbiosis}

Yael Haberman ${ }^{1,2}$, Melanie Schirmer ${ }^{3,4}$, Phillip J. Dexheimer ${ }^{1}$, Rebekah Karns ${ }^{1}$, Tzipi Braun ${ }^{2}$, Mi-Ok Kim ${ }^{1}$, Thomas D. Walters ${ }^{5}$, Robert N. Baldassano ${ }^{6}$, Joshua D. Noe ${ }^{7}$, Joel Rosh ${ }^{8}$, James Markowitz ${ }^{9}$, Wallace V. Crandall ${ }^{10}$, David R. Mack ${ }^{11}$, Anne M. Griffiths ${ }^{5}$, Melvin B. Heyman ${ }^{12}$, Susan S. Baker ${ }^{13}$, Richard Kellermayer ${ }^{14}$, Dedrick Moulton ${ }^{15}$, Ashish S. Patel ${ }^{16}$, Ajay S. Gulati ${ }^{17}$, Steven J. Steiner ${ }^{18}$, Neal LeLeiko ${ }^{19}$, Anthony Otley ${ }^{20}$, Maria Oliva-Hemker ${ }^{21}$, David Ziring ${ }^{22}$, Barbara S Kirschner ${ }^{23}$, David J. Keljo ${ }^{24}$, Stephen L. Guthery ${ }^{25}$, Stanley A. Cohen ${ }^{26}$, Scott Snapper ${ }^{27}$, Jonathan Evans ${ }^{28}$, Marla Dubinsky ${ }^{29}$, Bruce Aronow ${ }^{1}$, Jeffrey S. Hyams ${ }^{30}$, Subra Kugathasan ${ }^{31}$, Curtis Huttenhower ${ }^{3,4}$, Ramnik J. Xavier ${ }^{3,32}$ and Lee A. Denson ${ }^{1}$

Age-of-diagnosis associated variation in disease location and antimicrobial sero-reactivity has suggested fundamental differences in pediatric Crohn Disease (CD) pathogenesis. This variation may be related to pubertal peak incidence of ileal involvement and Peyer's patches maturation, represented by IFNY-expressing Th1 cells. However, direct mucosal evidence is lacking. We characterize the global pattern of ileal gene expression and microbial communities in 525 treatment-naive pediatric CD patients and controls $(\mathrm{Ctl})$, stratifying samples by their age-of-diagnosis. We show a robust ileal gene signature notable for higher expression of specific immune genes including GM-CSF and INFY, and reduced expression of antimicrobial Paneth cell a-defensins, in older compared to younger patients. Reduced a-defensin expression in older patients was associated with higher IFNY expression. By comparison, the CD-associated ileal dysbiosis, characterized by expansion of Enterobacteriaceae and contraction of Lachnospiraceae and Ruminococcaceae, was already established within the younger group and did not vary systematically with increasing age-ofdiagnosis. Multivariate analysis considering individual taxa, however did demonstrate negative associations between Lachnospiraceae and IFN $\gamma$, and positive associations between Bacteroides and a-defensin expression. These data provide evidence for maturation of mucosal Th1 immune responses and loss of epithelial antimicrobial a-defensins which are associated with specific taxa with increasing age-of-diagnosis in pediatric CD.

Mucosal Immunology (2019) 12:491-502; https://doi.org/10.1038/s41385-018-0114-4

\section{INTRODUCTION}

Epidemiologic associations, clinical phenotype, and natural history differ across CD age-of-diagnosis. ${ }^{1-4}$ Older children, adolescents, and young adults develop $C D$ involving the ileum most commonly, ${ }^{5}$ whereas colon-only involvement is predominant in the first decade of life and in the elderly., ${ }^{1,3}$ Variation in antimicrobial serology with increasing age-of-diagnosis within pediatric $C D$ has suggested potential age-dependent differences in the ileal microbial community and/or immune responses. ${ }^{6}$ These observations have informed the Paris classification system ${ }^{6}$ that sub-divides the Montreal A1 (0-16 years) classification for CD into diagnosis prior to age 10 years (A1a) and age 10-16 years (A1b). Recently, the younger A1a group was subdivided further to children diagnosed prior to 6 years of age, the very early onset type ${ }^{7,8}$ that was linked to monogenic forms of Inflammatory Bowel Disease (IBD) that differ from the polygenic form of CD diagnosed in older ages. ${ }^{4,9}$ Whether there was a specific mucosal basis for the clinical-based classification of the polygenic CD to younger A1a (6 years to $<10$ years) and older A1b (10-16 years) was not known.

The gut-associated lymphoid Peyer's patches (PP), concentrated $(50 \%)$ in the distal ileum $(\mathrm{TI}){ }^{10,11}$ interact with the overlying epithelium to induce tolerance or defense against luminal

\footnotetext{
${ }^{1}$ Cincinnati Children's Hospital Medical Center and the University of Cincinnati College of Medicine, Cincinnati, OH, USA; ${ }^{2}$ Sheba Medical Center, Tel-HaShomer, affiliated with the Tel-Aviv University, Tel-Aviv, Israel; ${ }^{3}$ The Broad Institute of MIT and Harvard, Cambridge, MA, USA; ${ }^{4} \mathrm{Harvard}$ T.H. Chan School of Public Health, Boston, MA, USA; ${ }^{5} \mathrm{Hospital}$ for Sick Children, University of Toronto, Toronto, ON, Canada; ${ }^{6}$ The Children's Hospital of Philadelphia, Philadelphia, PA, USA; ${ }^{7}$ Medical College of Wisconsin, Milwaukee, WI, USA; ${ }^{8}$ Goryeb

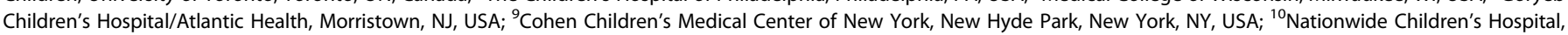
Columbus, OH, USA; ${ }^{11}$ Children's Hospital of Eastern Ontario, University of Ottawa, Ottawa, ON, Canada; ${ }^{12}$ University of California San Francisco, San Francisco, CA, USA; ${ }^{13}$ University at Buffalo, Buffalo, NY, USA; ${ }^{14}$ Texas Children's Hospital, Baylor College School of Medicine, Houston, TX, USA; ${ }^{15}$ Vanderbilt Children's Hospital, Nashville, TN, USA; ${ }^{16}$ UT Southwestern Medical Center at Dallas, Dallas, TX, USA; ${ }^{17}$ University of North Carolina, Chapel Hill, NC, USA; ${ }^{18}$ Riley Children's Hospital, Indiannapolis, IN, USA; ${ }^{19}$ Hasbro Children's Hospital, Providence, RI, USA; ${ }^{20}$ IWK Health Centre, Halifax, NS, Canada; ${ }^{21}$ John Hopkins University, Baltimore, MD, USA; ${ }^{22}$ Cedars-Sinai Medical Center, Los Angeles, CA, USA; ${ }^{23}$ University of Chicago Comer Children's Hospital, Chicago, IL, USA; ${ }^{24}$ Children's Hospital of Pittsburgh of UPMC, Pittsburgh, PA, USA; ${ }^{25}$ University of Utah, Salt Lake City, UT, USA; ${ }^{26}$ Children's Center for Digestive HealthMedicine, Atlanta, GA, USA; ${ }^{27}$ Children's Hospital - Boston, Boston, MA, USA; ${ }^{28}$ Nemours Children's Clinic, Jacksonville, FL, USA;

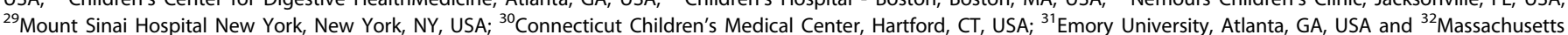
General Hospital, Harvard Medical School, Boston, MA, USA

Correspondence: Yael Haberman (Yael.haberman@cchmc.org)
}

Received: 15 June 2018 Revised: 6 November 2018 Accepted: 15 November 2018 Published online: 12 December 2018 
Table 1. Clinical and Demographic Characteristics stratified by age-of-diagnosis of the RISK cohort

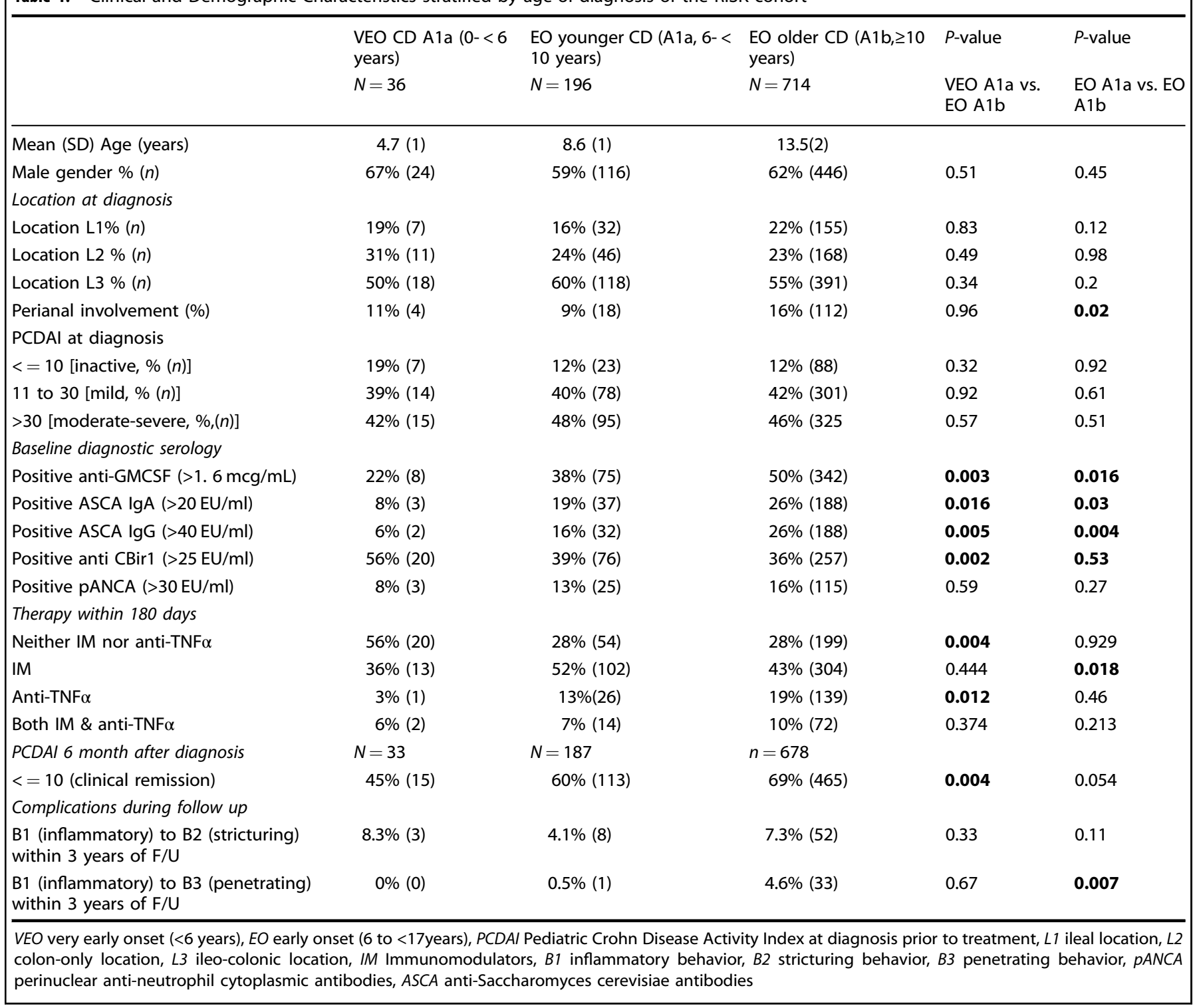

antigens. ${ }^{12}$ Importantly, PP undergoes dynamic maturation from birth, whereby the number and size of PP increase up to the second/third decade of life and then decline with age. The spatiotemporal relationship between the peak incidence of ileal CD (iCD) and PP evolution is represented primarily by INFYexpressing Th1 cells; this has led to the hypothesis that this dynamic mucosal immune process plays a central role in $\mathrm{iCD}$ pathogenesis., 50,11 In fact, murine studies have revealed maturation of an IFN- $\gamma$ associated immune signature in adult vs. preweaned mice, ${ }^{13}$ and a prior study in healthy children showed a pronounced Th1 polarization (increased IFNy and IL-12) within PP and adjacent ileal mucosa in response to bacterial antigens. ${ }^{14}$ However, comprehensive human mucosal-based gene expression analysis to test for maturation of a Th1 signature across the pediatric age-of-diagnosis range within $C D$ had not been performed.

Paneth cells, located at the base of the intestinal crypts, produce antimicrobial peptides such as lysozymes and adefensins to modulate the intestinal microbiota and are an important arm of the innate immune response. ${ }^{15}$ Reduced human a-defensins (DEFA5 and DEFA6) expression were previously documented in patients with iCD. ${ }^{16}$ However, it is unclear if this deficiency is a primary event in $\mathrm{iCD}$ or a secondary event occurring as a consequence of inflammation. ${ }^{17}$ Whether differences in $a-$ defensin expression would be observed as a function of age-ofdiagnosis in pediatric $\mathrm{iCD}$, and whether this in turn is associated with alterations in the microbial community, is not known. Interestingly, our group has recently shown that in a competingrisk model, older age-of-diagnosis, African American race, and ASCA IgA and CBir1 sero-positivity were associated with internal penetrating (B3) disease complications. ${ }^{18}$ To improve our understanding of pediatric CD pathogenesis across different ages-ofdiagnosis, we employed combined ileal mRNA and 16s rRNA sequencing approaches to define host transcriptome and microbial community differences in patients stratified by their age-ofdiagnosis. We show that despite an age-independent microbial dysbiosis in pediatric $C D$, there are robust differentially expressed host ileal gene signature differences with higher expression of inflammatory-related genes, and a lower expression of Paneth cell a-defensins, with increasing age-of-diagnosis. 
Table 2. Clinical and demographic characteristics

\begin{tabular}{|c|c|c|c|c|c|}
\hline & $\mathrm{Ctl} n=50$ & $\mathrm{cCD} n=56$ & $\mathrm{iCD} n=198$ & $\mathrm{iCD}$ A1a $n=48$ & $\mathrm{iCD} \mathrm{A} 1 \mathrm{~b} n=150$ \\
\hline Male gender (\%) & 62 & 59 & 63 & 67 & 59 \\
\hline Perianal involvement (\%) & - & 21 & 16 & 10 & 17 \\
\hline Ileal deep ulcers (\%) & - & - & 45 & 45 & 46 \\
\hline 11 to 30 (mild, \%) & - & 48 & 52 & 46 & 53 \\
\hline$>30$ (moderate-severe, \%) & - & 46 & 39 & 42 & 39 \\
\hline
\end{tabular}

\section{MATERIALS AND METHODS}

The RISK cohort

The Crohn's and Colitis Foundation sponsored RISK prospective inception cohort study ${ }^{18-20}$ included newly diagnosed pediatric CD patients (Table 1) at 28 North American pediatric gastroenterology centers. 946 patients had inflammatory (B1) disease behavior and no disease complications (B2 or B3) at the time of diagnosis. All patients were required to undergo baseline colonoscopy and confirmation of characteristic endoscopic features and chronic active colitis/ileitis by histology prior to diagnosis and treatment, and documented initial clinical severity and disease location. Non-IBD controls were subjects suspected to have IBD, but with normal radiographic, endoscopic, and histologic findings. Ileal biopsy samples from a CD sub-cohort representative of the overall RISK cohort (age, gender, and disease phenotype and severity) and non-IBD controls (age and gender) are included in our mucosal mRNAseq analysis (254 CD and 50 control (Ctl), Tables 1, 2). lleal biopsies of CD patients $(n=272)$ and $\mathrm{Ctl}(n=178)$ were also analyzed for ileal microbial profiles and were already included in our recent reports, ${ }^{18-20}$ of those 197 also had mRNAseq $(160 \mathrm{CD}$ and $37 \mathrm{Ctl})$. Patient-preparation for endoscopy and treatment course were according to the dictates of their physicians, not by standardized protocols. Serological determination of perinuclear anti-neutrophil cytoplasmic antibodies (pANCA), anti-Saccharomyces cerevisiae antibodies (ASCA) $\operatorname{lgG}$, ASCA IgA, and anti-CBir1, was performed at Cedars-Sinai Hospital (Los Angeles, CA, USA). ${ }^{21}$ Granulocyte-macrophage colony-stimulating factor (GM-CSF) autoantibodies were measured at Cincinnati Children's Hospital Medical Center (Cincinnati, $\mathrm{OH}$, USA). Positive serologies were defined based on specific predefined cut points. anti-GMCF is consider positive with values above $1.6 \mathrm{mcg} / \mathrm{mL}^{22}$ the value previously reported within pediatric-onset $C D$ patients linked to higher risk of stricturing disease complications, and subsequently validated within adultonset $C D$ patients for this outcome. Other antibody levels were determined and results are expressed as ELISA units (EU/ml), which are relative to a Cedars-Sinai Laboratory standard, derived from a pool of $C D$ patient sera with well-characterized disease found to have reactivity to this antigen; ASCA $\lg \mathrm{A}$ is considered positive above $20 \mathrm{EU} / \mathrm{ml}$, ASCA lgG above $40 \mathrm{EU} / \mathrm{ml}$, anti-CBir1 above $25 \mathrm{EU} / \mathrm{ml}$, and pANCA above $30 \mathrm{EU} / \mathrm{ml}$.

Ileal DNA and RNA extraction and RNA-seq

DNA and RNA were isolated from ileal biopsies as previously described..$^{19}$ Reads were quantified by kallisto, ${ }^{23}$ using Gencode v23 as the reference genome and Transcripts per Million (TPM) as an output. We included 13,206 protein-coding genes with TPM above 5 in 5 samples. Differentially expressed genes were determined in GeneSpring ${ }^{\circledR}$ software with fold change differences $(F C)>=1.5$ and using false discovery rate correction (FDR $<0.05)$. Euclidean distance metric and Ward's linkage rule was used for unsupervised hierarchical clustering. ToppGene ${ }^{24}$ and ToppClus$\operatorname{ter}^{25}$ software were used to test for functional annotation enrichment analyses. Visualization of the network was obtained using Cytoscape.v3.0.2. ${ }^{26}$ The RNASeq data is deposited in the GEO repository (GSE101794).

Microbial community profiling and analysis of associations testing between microbial taxa and clinical and molecular metadata Detailed protocols used for $16 \mathrm{~s}$ rRNA amplification and sequencing are as previously described. ${ }^{18,20,27}$ In brief, 16 s rRNA amplicon sequencing of 450 ileal samples was performed using the Illumina MiSeq v2 platform, targeting the V4 region of the SSU rRNA gene, and generating paired-end reads of $175 \mathrm{~b}$. Samples with at least 3000 reads were included. Taxonomy was assigned based on the Greengenes database. ${ }^{28}$ Each OTU was required to occur at a relative abundance of at least $0.01 \%$ across all samples and be present in at least $5 \%$.

Differentially abundant taxa were determined based on multivariate statistical analyses using Multivariate Analysis by Linear Models (MaAsLin). ${ }^{18-20}$ The following metadata were investigated in the analysis: age, gender, body mass index (BMI, as a measure of nutritional status), clinical phenotype (Ctl or CD), endoscopic severity (deep ulcers in ileum), clinical severity (Pediatric Crohn's Disease Activity Index, PCDAl), ileal gene expression of CSF2, CXCR1, IFNG, MMP3, DEFA5, GSTA1, and LCT, and NOD2 and ATG16L1 IBD risk allele carriage. Significant association was considered below a $q$ value (Benjamini-Hochberg) threshold of 0.2 .

\section{Immunohistochemistry}

Immunohistochemistry detection of the human alpha-defensin five protein encoded by the DEFA5 gene in control and CD ileal biopsies was performed as previously described in the CCHMC Digestive Healthy Center core facility, ${ }^{19}$ using anti-DEFA5 (mouse monoclonal anti-human alpha-defensin NP5 Ab, clone $\mathrm{nr} 8 \mathrm{C} 8$, code ab62757, Abcam, Cambridge, UK). Staining was examined using an Olympus BX51 light microscope and digitally recorded at $\times 20$ and $\times 40$ magnification.

Ethical considerations

The institutional review board reviewed and approved the protocol and informed written consent was obtained. ClinicalTrials.gov identifier is NCT00790543. 
a

b
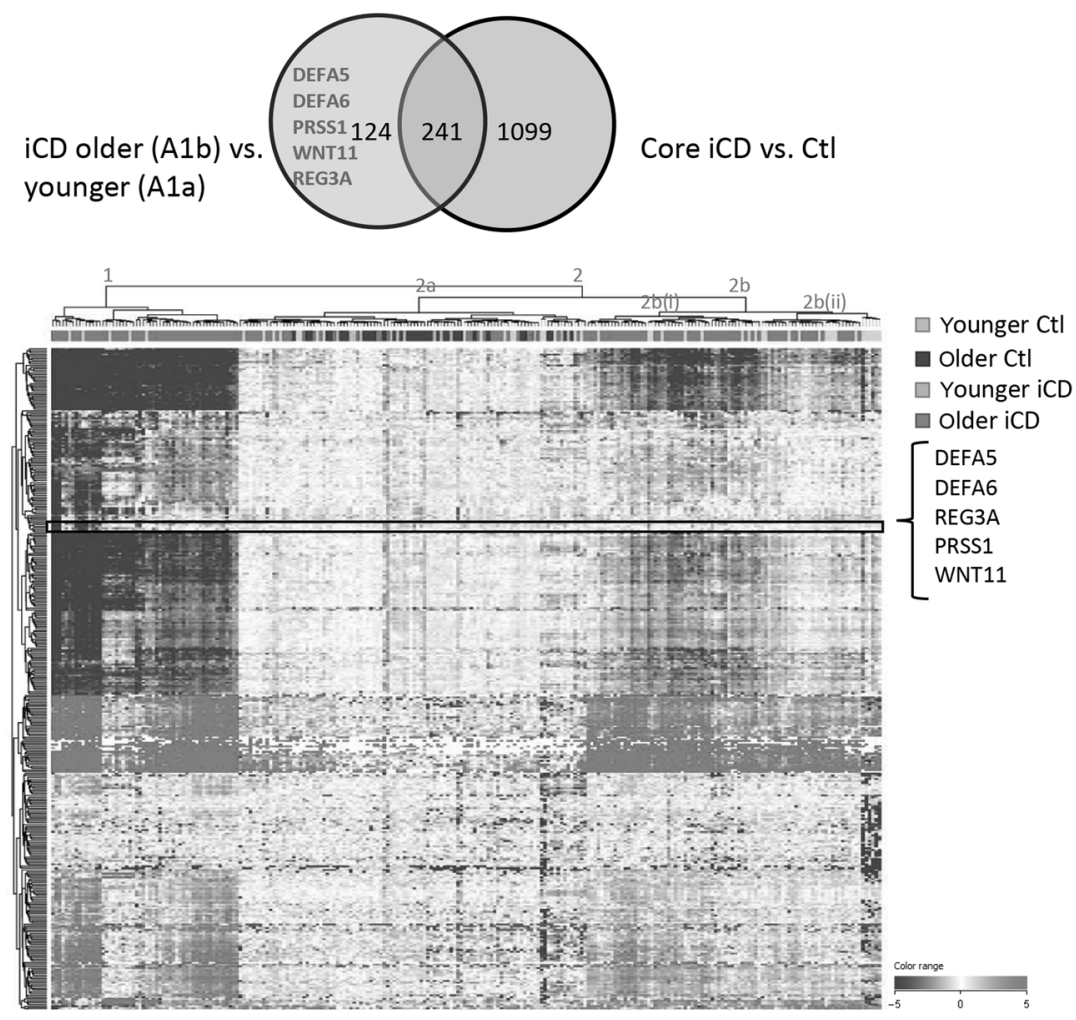

c

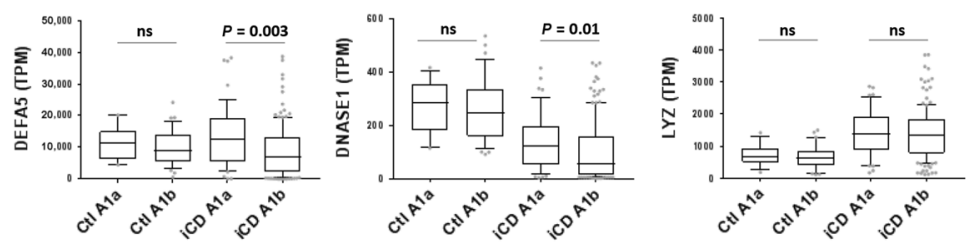

d
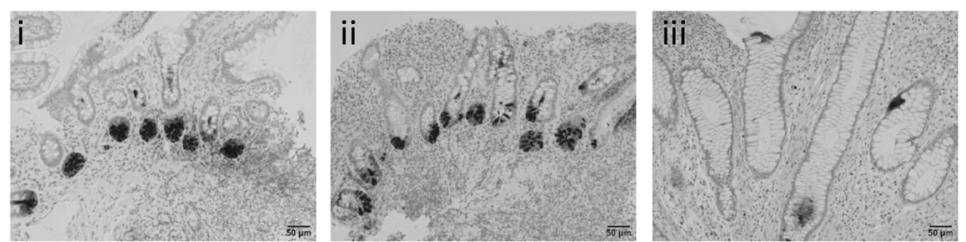

e Age-of-onset (years)

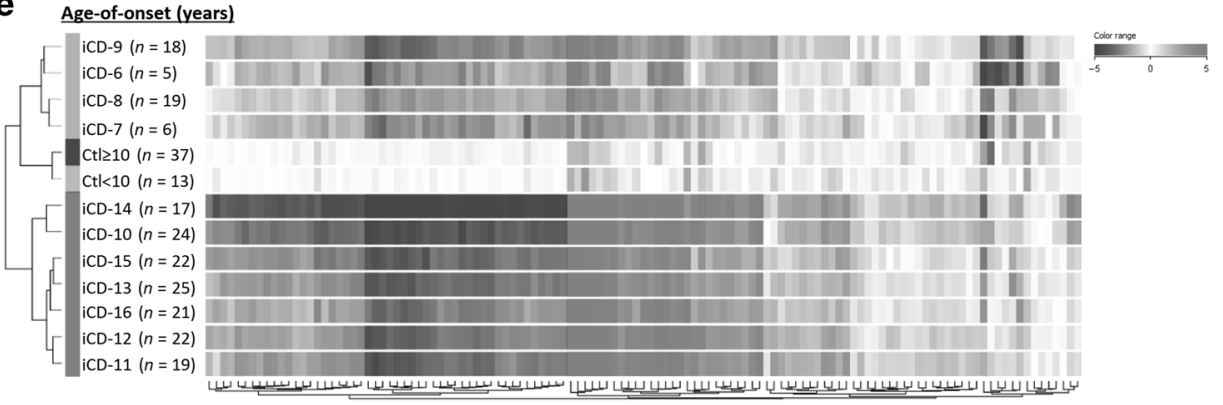

\section{RESULTS}

The RISK cohort

RISK is a prospective inception cohort study which enrolled pediatric CD patients at diagnosis at 28 sites in North America. RISK includes 946 treatment naïve newly diagnosed CD patients that were all classified as having an inflammatory B1 phenotype without B2 (stricturing/narrowing) or B3 (internal penetrating behavior) complications at diagnosis. Change in disease behavior from B1 inflammatory to either B2 stricturing or B3 penetrating behavior was recorded during follow up. For the purpose of our study, patients were stratified based on the Paris age-of-diagnosis classification, where diagnosis 
Fig. 1 Decreased epithelial Paneth cell $\alpha$-defensins signature in pediatric iCD $\geq 10$ years at diagnosis. a Venn diagram shows an overlap between the previously reported 1340 core iCD gene signature ${ }^{19}$ and the 365 genes that were differentially expressed in the ileum between older (A1b) and younger (A1a) iCD (FDR correction [0.05], fold-change $\geq 1.5$ ). 124 of these 365 genes including $\alpha$-defensins and REG3A were not included in the core iCD gene list. $\mathbf{b}$ Unsupervised hierarchical clustered heat map of the 365 genes differentially expressed genes between older and younger iCD with upregulated genes in red and down-regulated genes in blue. Above the heat map, younger Ctl (light blue), older $\mathrm{Ctl}$ (dark blue), younger CD (orange), and older CD (red) samples are indicated. c TPM ileal gene expression is shown for the indicated genes for the indicated groups stratified by age-of-diagnosis with Kruskal-Wallis test with Dunn's multiple comparisons. $\mathbf{d}$ Immunohistochemistry in representative CD patients and non-IBD controls. Relatively high DEFA5 staining is shown for older non-IBD control (i) and younger CD (ii) that correlated with transcripts per million (TPM) values of $>10,000$ and 6143 for gene expression by RNASeq, respectively, and relatively low DEFA5 staining in older CD that correlated with transcripts per million (TPM) values of 1239 for gene expression by RNASeq. Images were captured using an Olympus BX51 light microscope and digitally recorded at $\times 20$ magnification. e Averaged and unsupervised hierarchical clustering heat map of 121 of 365 (top third differentially expressed genes between older and younger iCD) stratified by yearly age-of-diagnosis as indicated. Number of patients for each age group is indicated adjacent to the heat map

prior to age 10 years is defined as $A 1 a$, and age $10-16$ years is defined as $\mathrm{A} 1 \mathrm{~b}$. The younger A1a were further subdivided to very early onset (VEO, <6 years $\left.{ }^{7}\right)$, and to early onset (EO) (6-9 years).,

Table 1 shows demographic and clinical characteristics categorized by age-of-diagnosis. Of the $946 \mathrm{CD}$ patients in the RISK cohort, only $36(4 \%)$ were classified as VEO (<6 years) CD, 196 (21\%) as EO younger CD (6-9 years), and the rest (75\%) as EO older CD (10-16 years). Clinical severity defined using the Pediatric Crohn's Disease Activity Index (PDCAl) was not significantly different between the groups at diagnosis. Perianal involvement was significantly higher in older CD. As previously reported, ${ }^{6}$ seropositivity for anti-GMCSF and ASCA were significantly increased with age-of-diagnosis classifications. There were no significant differences in baseline PCDAl, early anti-TNF exposure that was previously associated with lower rate of penetrating (B3) complications, ${ }^{18}$ and PCDAl six months after diagnosis between older and younger CD cases. However, there was a significantly higher prevalence of penetrating complication (B3) during 3 years follow up in older vs. younger CD (Table 1).

Based on those differences between older (10-16 years) and younger EO CD, the relative low number of VEO cases, and the likelihood association between the VEO cases and the monogenic IBD type, we included hereafter only patients older than 6 years in the mucosal transcriptomics and microbial analyses. Ileal mucosal transcriptomics and microbial characterization were done on a representative sub-group of ileal CD ( $\mathrm{C} C \mathrm{D}, n=198)$, colon only $C D$ ( $C C D n=56)$, and non-IBD CtI $(n=50)$ (Table 2 and Suppl. Table 1). CCD patients are subjects who met diagnostic criteria for $C D$ but lacked ileal inflammation on endoscopy. This unique large patient population and biospecimens offered an opportunity to directly test for the association between the presence of mucosal inflammation, host mucosal genes and pathways, and mucosal microbial composition by performing an age-dependent analyses in treatment-naive patients.

Decreased expression of a-defensins in older iCD

We identified 365 genes (Suppl. Table 2 and Fig. 1) that were significantly differentially expressed between older and younger iCD. We have previously reported the identification of a core iCD gene expression signature. ${ }^{19}$ Interestingly, the a-defensin genes (DEFA5 \& DEFA6) and REG3A were not included in the core iCD list (Fig. 1a), as these were only suppressed in older iCD compared to both younger $\mathrm{iCD}$ and $\mathrm{Ctl}$ (Fig. 1c). Results of unsupervised clustering using the 365 differentially expressed genes are shown in Fig. $1 \mathrm{~b}$ with the indicated location of a-defensins. 51 of 148 (34.5\%) older CD and 5 of 48 (10.4\%) younger CD (Chi-squares $p=$ 0.0013 ) are in block 1, while 20 of $148(13.5 \%)$ older, and 16 of 48 (33\%) younger $C D$ (Chi-squares $p=0.002$ ) are in block $2 b(i i)$. DNASE1 that was negatively associated with abnormal Paneth cell morphology in $\mathrm{CCD}^{29}$ showed a similar pattern of lower expression level in older $\mathrm{iCD}$ as the a-defensins and $R E G 3 G / A$ in contrast to stable increased expression of lysozyme ( $L Y Z$, Fig. 1c and Suppl. Table 2). Consistent with this, a reduced frequency of alpha- defensin 5 positive epithelial cells were detected in ileal biopsies from older $C D$ patients, compared to younger $C D$ patients and controls (Fig. 1d). Unsupervised hierarchical clustering analysis identified groups of biopsies with similar ileal gene expression profiles. Unsupervised clustering using the top third most differentially expressed genes subset (121 of 365 genes) demonstrated that patients diagnosed at age 6, 7, 8, and 9 years clustered together and separately from patients who were diagnosed at age 10,11, 12, 13, 14, 15, and 16 years (Fig. 1e and suppl. Figure 1).

Enhanced ileal immune responses in older iCD

We next tested whether the ileal transcriptome of older iCD exhibited mucosal immunologic maturation represented by a Th1 gene expression profile compared with younger iCD. Functional annotation enrichment analyses using ToppGene ${ }^{24}$ and ToppClus$\operatorname{ter}^{25}$ were used to map groups of related genes within the 365 gene signature to biologic functions and immune cell types (Fig. 2a, Suppl. Table 3 and 4). Genes up-regulated in older iCD were notable for higher expression of an inflammatory-related signature including GM-CSF (CSF2), IFNY, matrix metalloproteinases, and collagens (Suppl. Table 2). This gene signature showed functional annotation enrichment (Fig. 2a and Suppl. Table 4) for genes induced in response to molecules of bacterial origin $(P<$ $4.82 \mathrm{E}-07$ ) including several monocyte-derived pro-inflammatory cytokines and the Th1-related cytokine IFN $\gamma$. Additional functional annotation enrichment was noted for genes expressed by granulocytes $(P<2.65 \mathrm{E}-06)$, B cells $(P<2.72 \mathrm{E}-04)$, and lymphoid stromal cells $(P<8.1 \mathrm{E}-14$, Fig. $2 \mathrm{~b})$. The upregulated genes also showed a remarkable increase for genes encoding extracellular matrix (ECM) and extracellular matrix-associated proteins $(P<$ $4.95 \mathrm{E}-16)$, extracellular space $(P<1.12 \mathrm{E}-26)$, and genes defining epithelial-mesenchymal transition $(P<4.51 \mathrm{E}-15)$. The functional annotation enrichment analyses included enrichment for genes associated with a specific drug or supplement, with one of the top enrichments for curcumin ( $P<1.15 \mathrm{E}-9$, Suppl. Table 4). We also noted enrichment for collagen genes $(P<1.05 \mathrm{E}-05)$ including COL12A1, COL1A1, COL3A1, COL6A3, COL7A1, and COL8A1. Of note, this age-dependent gene signature was specific, as several other mucosal inflammatory genes including the $\mathrm{NADPH}$ oxidase DUOX2, FOLH1, REG1A, and SAA2, that we previously identified as part of a core $i C D$ gene signature, ${ }^{19}$ were upregulated to the same degree in older and younger $\mathrm{iCD}$ and are not included in the 365 gene list. These results defined a maturation of components of both the innate and Th1 adaptive ileal gene signature in pediatric CD patients with older age-of-diagnosis.

A similar approach was applied to the downregulated genes between older and younger iCD (Fig. 2b). These analyses identified decreased genes associated with brush border localization $(P<$ $5.23 \mathrm{E}-24)$, digestion $(P<1.65 \mathrm{E}-13)$, lipid metabolic processes $(P$ $<1.58 \mathrm{E}-08)$, and vitamin digestion and absorption $(P<1 \mathrm{E}-07)$ in older iCD. Additionally, we found that 32 genes of the 365 genes had a FC $\geq 1.5$ in older vs younger Ctl (Suppl. Table 5), including 


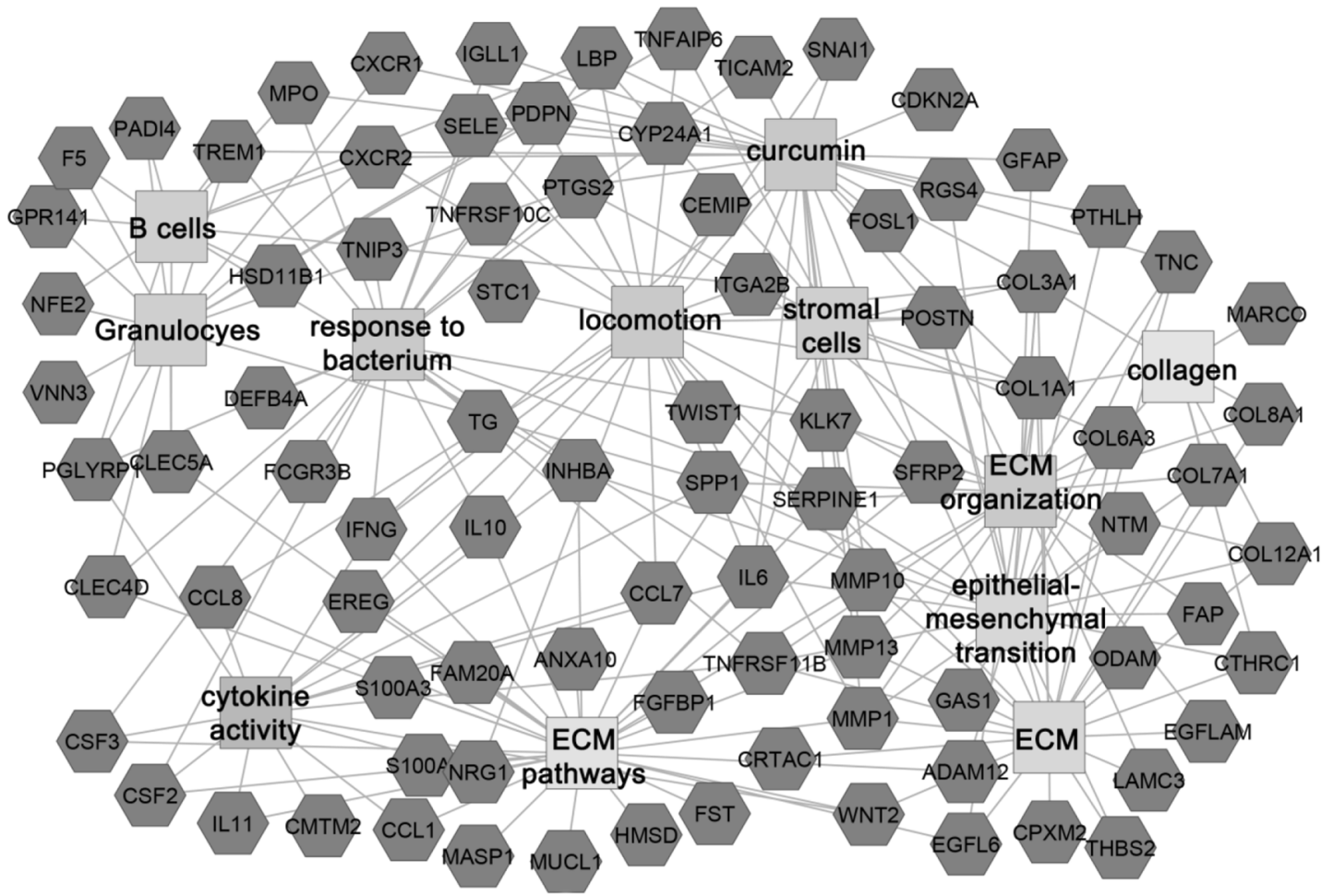

b

Downregulated genes in older ( $A 1 b \geq 10$ years) vs. younger ( $A 1 a<10$ years) $\mathrm{iCD}$ patients

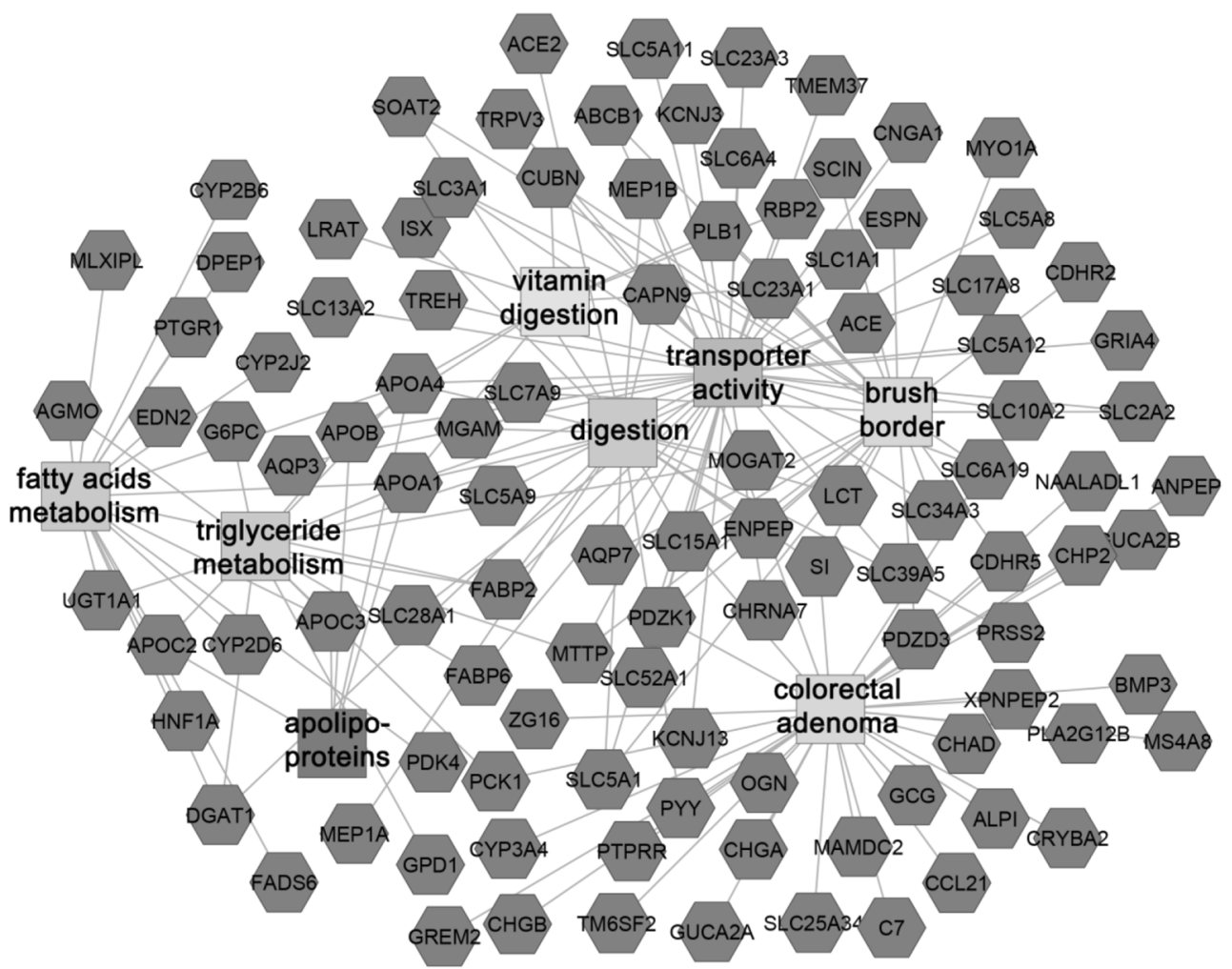

Fig. 2 Enhanced ileal innate and adaptive Th1 immune responses in pediatric iCD $\geq 10$ years at diagnosis. a Top functional annotation enrichment analyses using Toppgene/ToppCluster ${ }^{25}$ platforms of the 171 upregulated and differentially expressed genes between older and younger iCD and Cytoscape ${ }^{26}$ was used for visualization. b Top functional annotation enrichment analyses using Toppgene/ToppCluster ${ }^{25}$ platforms using the 194 downregulated and differentially expressed genes between older and younger iCD and Cytoscape ${ }^{26}$ was used for visualization 


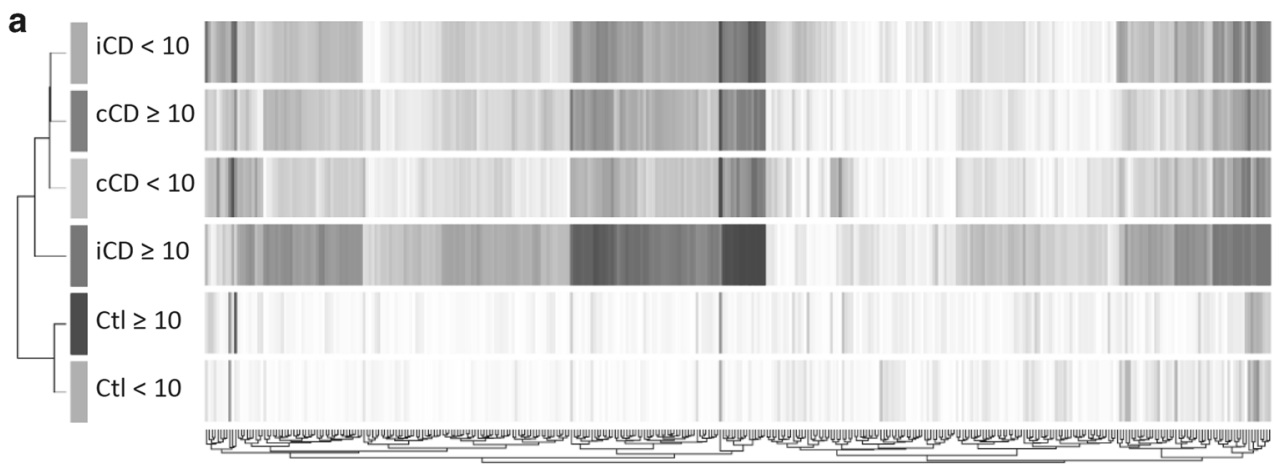

b

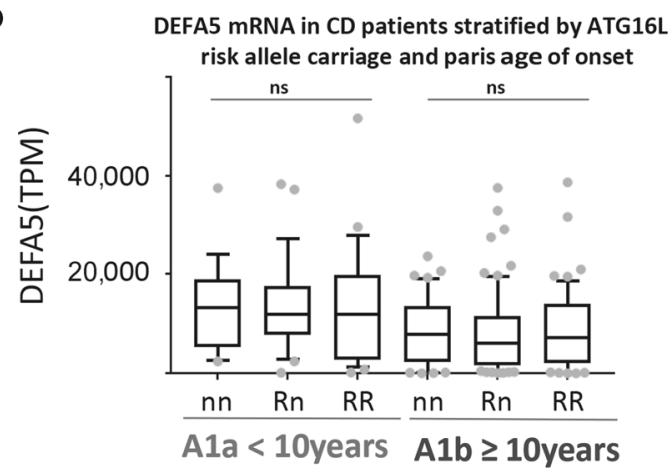

C

DEFA5 mRNA in CD patients stratified by NOD2 risk allele carriage and paris age of onset

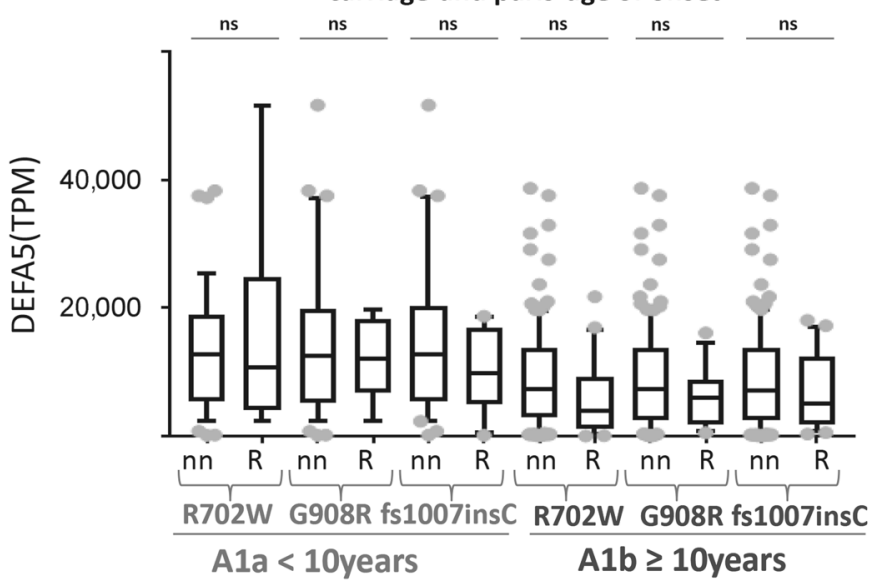

Fig. 3 Decreased epithelial Paneth cell signature in pediatric $C D \geq 10$ years at diagnosis is associated with clinical ileal inflammation. a Unsupervised averaged hierarchical clustered genes heat map of 365 genes differentially expressed between older and younger iCD is shown for each clinical sub-group [Ctl, cCD, and iCD divided to $<10$ and $\geq 10$ years]. b, c DEFA5 TPM ileal gene expression is shown for the indicated younger and older CD age-of-diagnosis groups as indicated, stratified by their ATG16L1 (b) or NOD2 (c) genotype. R; risk allele (homozygote or heterozygotes), nn; no risk allele, nR; heterozygote for the risk allele. RR; homozygote for the risk allele

$L C T$ that showed decreased expression $\geq 2$ in the older vs. younger groups. However, only 2 passed corrected $P$-values FDR of $\leq 0.05$.

Decreased a-defensins expression in older $C D$ is associated with Th1 IFNY and inflammation but not with NOD2 or ATG16L1 genotype

Our cohort also included CCD patients, who met diagnostic criteria for $C D$ but lacked ileal inflammation on endoscopy. This unique patient population offered an opportunity to directly test for the association between mucosal inflammation and gene expression by performing age-dependent analyses. Unsupervised clustering using specifically the 365 genes that were differentially expressed between older and younger iCD demonstrated that while patients groups first clustered based on disease phenotype (CD and $\mathrm{Ctl}$ ), older CCD clustered with younger $\mathrm{CCD}$ and $\mathrm{CCD}$ (Fig. 3a). a-defensin gene expression was not significantly decreased in the ileum of older $\mathrm{CCD}(\mathrm{A} 1 \mathrm{~b})$ in comparison to younger $\mathrm{CCD}$ or $\mathrm{Ctl}$ in a univariate non parametric $t$-test. 84 (Suppl. Table 6) of the 365 genes differentially expressed between older and younger iCD showed a FC $>1.5$ in the ileum of older vs. younger CCD including $D E F A 5$ but none passed the FDR $<0.05$. To capture differences between older CD with and without clinical ileal inflammation (iCD vs. $C(D)$, we identified 1135 genes that were significantly differentially expressed between older iCD and CCD (Suppl. Table 7). Those 1135 included CSF2 and IFNG that were upregulated in all $C D$ forms in comparison to $\mathrm{Ctl}$, with further increased expression in older iCD. Of note, no genes were differentially expressed between younger $\mathrm{i} C D$ and $C C D$, showing 

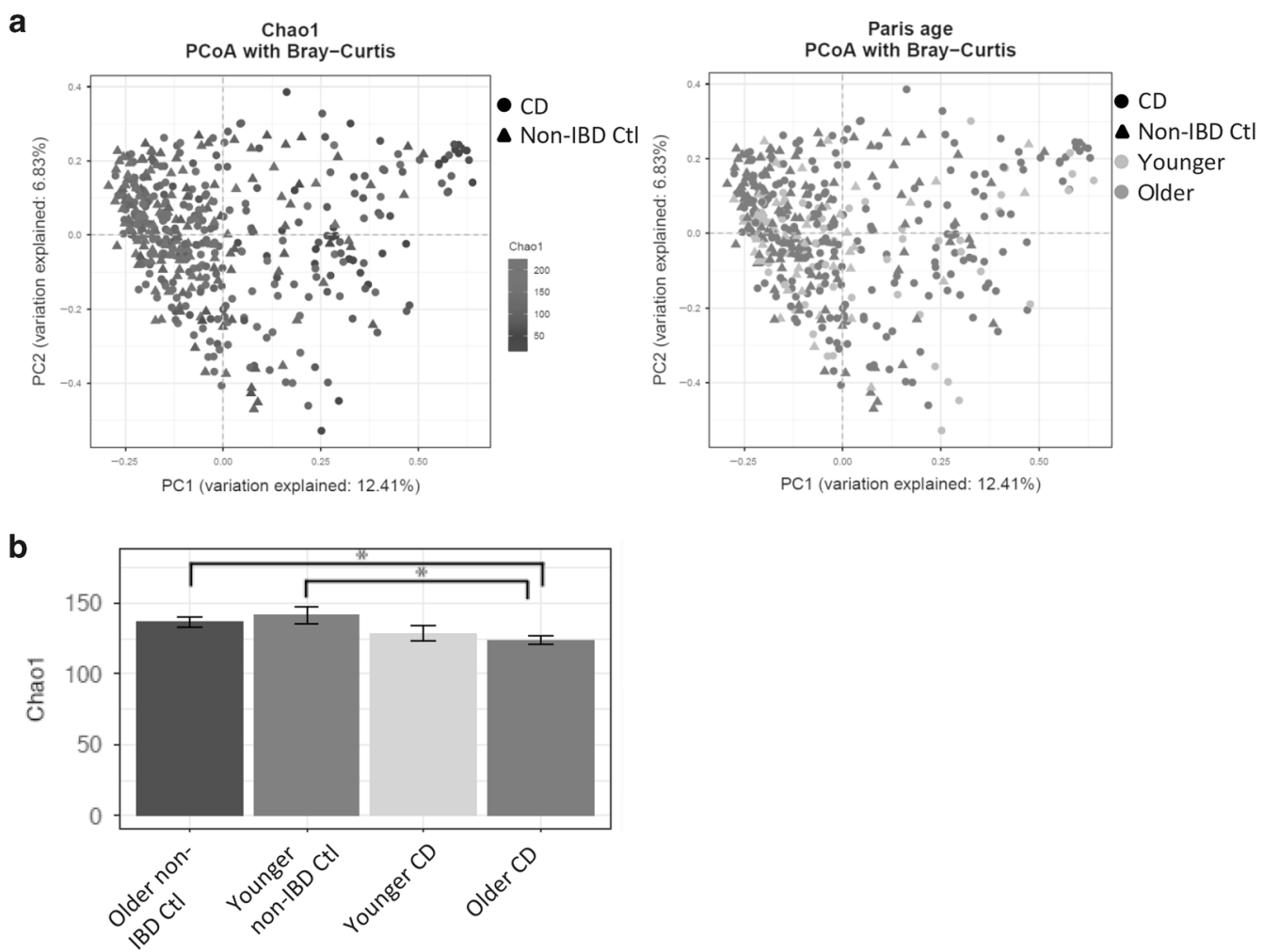

C

Mean non-IBD Ctl older/younger

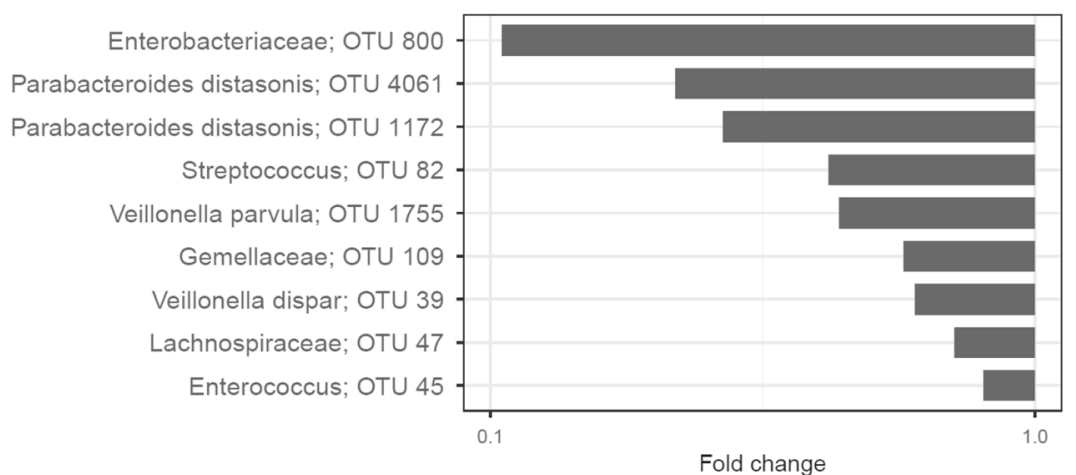

Fig. 4 Age-associated shifts in the ileal microbial community composition detected in non-IBD controls are not present within CD. a PCoA with Bray-Curtis distance comparing microbial community diversity in samples from CD patients $(n=272)$ and Ctl ( $n=178)$. Left panel, samples are colored by the Chao1 diversity index. Right panel, samples are colored by $<10$ and $\geq 10$ years. Triangular shape indicates Ctl, filled circles indicate CD samples. b Mean and standard deviation of Chao1 $\alpha$-diversity is shown for $\mathrm{Ctl}$ and $\mathrm{CD}$ divided in younger $<10$ and older $\geq 10$ years. ${ }^{*} P<0.01$, a two-sided $t$-test was used. $\mathbf{c}$ The bar graph shows fold change (mean older $\mathrm{A} 1 \mathrm{~b} \mathrm{Ctl} / \mathrm{mean}$ younger $\mathrm{Ctl}$ ) for significant associations between the indicated taxa as determined by MaAsLin while taking Paris age, gender, and body mass index (BMI) into account

that genes such as CSF2 and IFNG were associated with overt mucosal inflammation specifically in the older age group. We next asked whether a-defensin expression would be associated with NOD2 or ATG16L1 genotype. We did not observe a significant difference when we compared DEFA5 expression between patients stratified by ATG16L1 or NOD2 (Fig. 3b and c) risk allele carriage.

Th1-related IFNy was suggested to suppress human a-defensin gene expression, ${ }^{30}$ while a-defensin expression was shown to specifically inhibit Th1-related IFN $\gamma$ inflammation in a murine model. ${ }^{31}$ We identified significant negative associations between IFNG and DEFA5 gene expression (Pearson $r=-0.33, P<0.0001$ ) in our cohort. Collectively, these results characterized an unexpected age-dependent downregulation of a-defensin genes within pediatric $C D$ which was in turn associated with increasing
IFNG expression and intensified extracellular matrix and collagen expression in patient with older age-of-diagnosis.

Microbial dysbiosis in pediatric CD is already established in the younger group and lacks systematic changes observed in non-IBD Ctls

We have previously described a pediatric CD-associated ileal dysbiosis. ${ }^{19,20}$ Variation in microbial samples was compared by using Principal Coordinate Analysis (PCoA) with Bray-Curtis distance and samples were colored by either the Chao1 $a-$ diversity (richness within a sample) or Paris age (Fig. 4a). The frequency of younger and older CD with $\mathrm{PC} 1$ values $>0$ was not significantly different between the two groups $(41 \%$ of the younger $C D$ and $45 \%$ of the older $C D$ ). Differences in a-diversity were the main driver of variation rather than age or diagnosis, as 


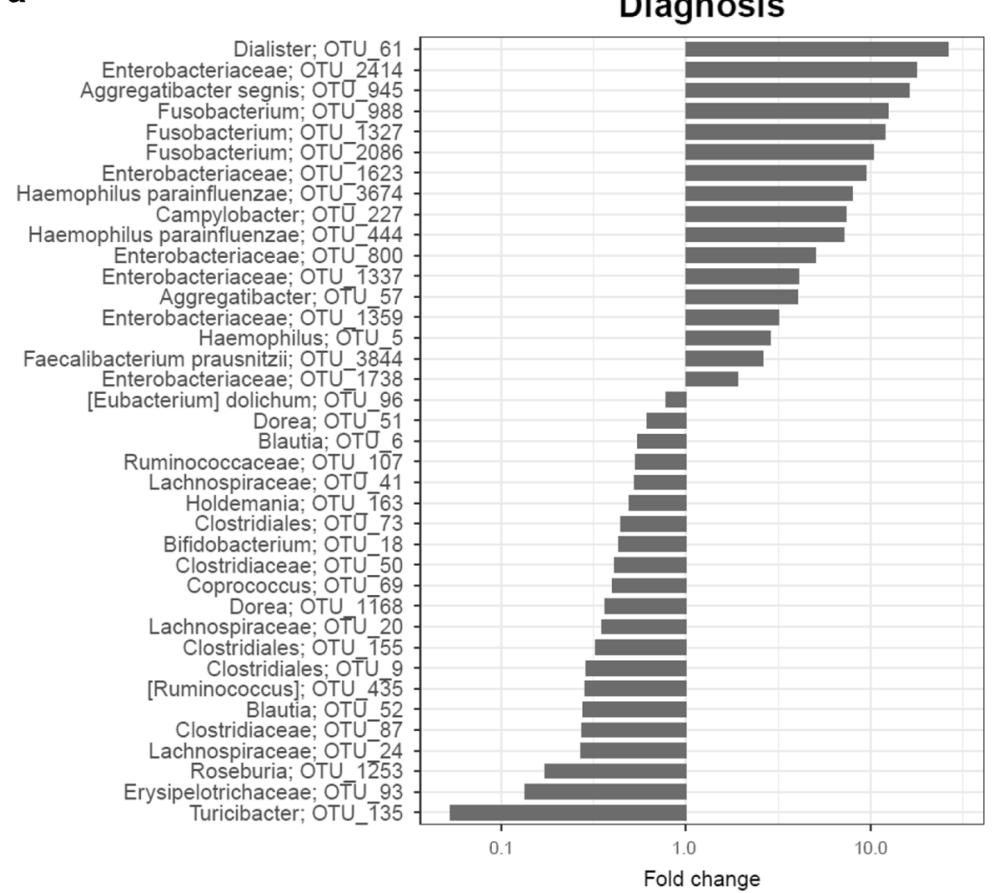

Mean CD/non-IBD CtI

b
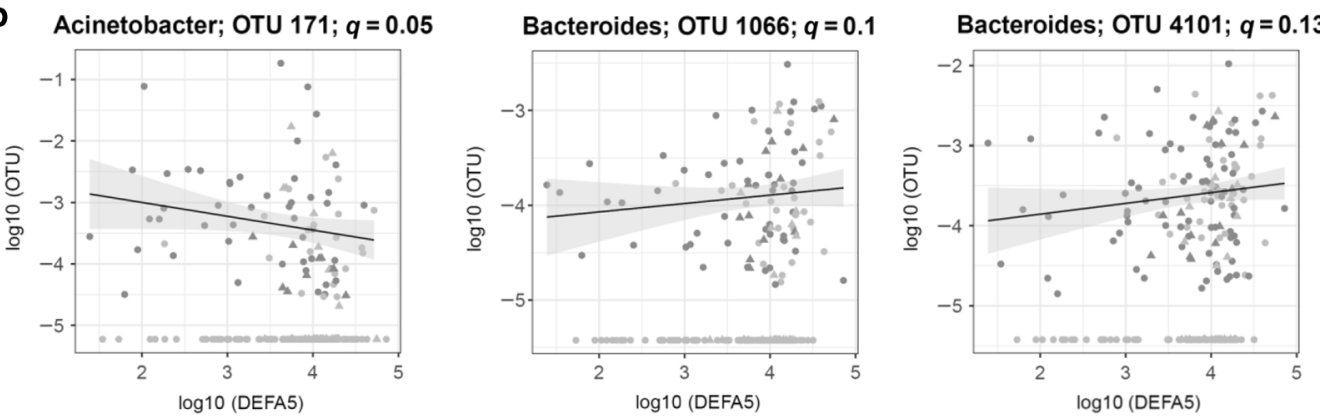

Bacteroides fragilis; OTU 755; $q=0.13$ Lachnospiraceae; OTU 612; $q=0.15$
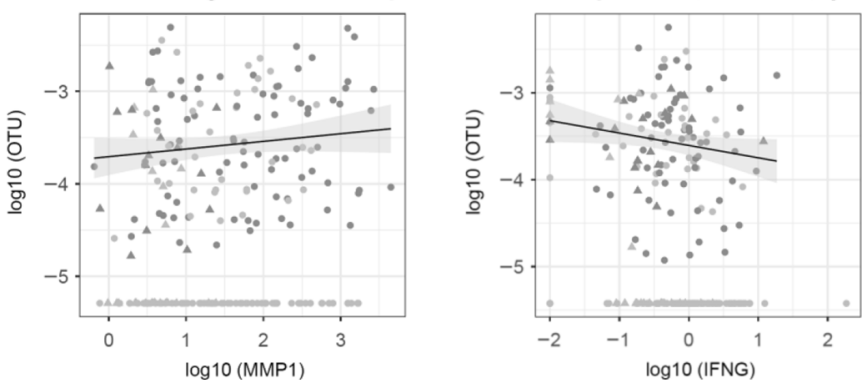

- $\mathrm{CD}$

- Non-IBD CtI

- Younger

- Older

- Org. not present

Fig. 5 Co-variation of the ileal microbial community structure with host gene expression. a The bar graph shows fold change (mean CD/mean $\mathrm{Ctl}$ ) for significant associations between the indicated taxa and clinical phenotype (Ctl, CD) as determined by MaAsLin while taking Paris age, gender, body mass index (BMI, as a measure of nutritional status), endoscopic severity (deep ulcers in ileum), clinical severity (Pediatric Crohn's Disease Activity Index, PCDAl), antibiotics, ileal gene expression of CSF2, CXCR1, IFNG, MMP3, DEFA5, GSTA1, and LCT, and NOD2, and ATG16L1 IBD risk allele carriage into account. b Scatter plots are shown for significant associations between the indicated taxa ( $y$-axis) and host gene expression ( $x$-axis) based on the multivariate statistical analysis described in a

was previously observed. Further, a-diversity was significantly reduced in older $C D(C D A 1 b)$ vs. Ctl of any age. To capture agedependent differences we applied a multivariate statistical framework (MaAsLin) ${ }^{20}$ to Ctl samples $(n=177, n=47$ for younger and $n=130$ for older) and CD samples $(n=272, n=64$ for younger and $n=208$ for older), respectively. We were able to detect significant differences in microbial abundances between older vs. younger $\mathrm{Ctl}$ ( $\geq 10$ years vs. $<10$ years) while controlling for body mass index (BMI), ethnicity, gender, and NOD2, and ATG16L1 risk allele carriage (Fig. 4C). This included a significant decrease of Enterobacteriaceae, Parabacteroides distasonis, Streptococcus, Veillonella, Gemellaceae, Lachnospiraceae, and Enterococcus in older Ctl. Applying the same analysis to CD samples, while additionally controlling for antibiotic usage and ileal deep ulcers yielded no 


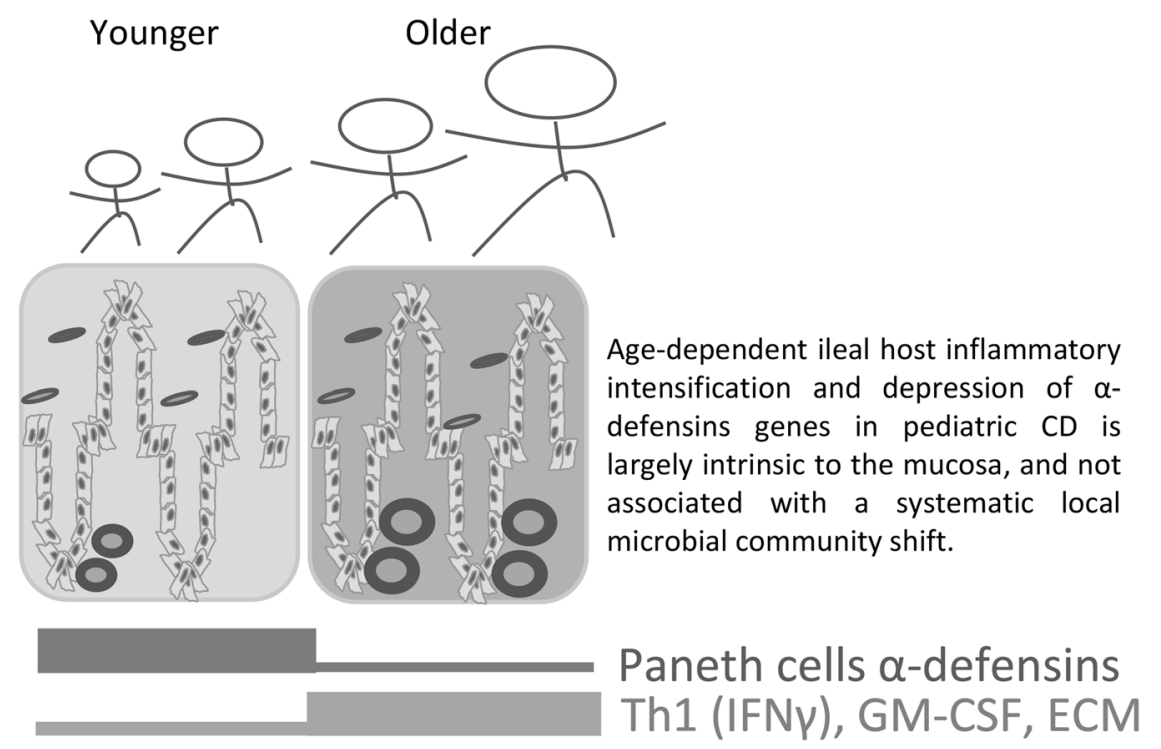

Fig. 6 Summarizing cartoon. Using high throughput mRNA and 16s rRNA amplicon sequencing of treatment naïve newly diagnosed ileal biopsies from $C D$ cases we identify 365 genes that were robustly differentially expressed between older iCD (10-16 years) and younger iCD $<10$ years). Those differentially expressed genes showed increased Th1-related IFN $\gamma$ profile associated with amplified innate myeloid inflammatory activation, and enhanced extracellular matrix and collagen signatures in older iCD cases. Remarkably, these signatures for enhanced immune activation was associated with a specific reduction in epithelial Paneth cell $\alpha$-defensin expression in older iCD, and was not associated with systematic changes in the local mucosal microbial communities

significant associations. Collectively these analyses demonstrated no systematic changes in CD across age-of-diagnosis suggesting that that $\mathrm{CD}$-associated dysbiosis was already established at diagnosis in the younger CD patients.

Host gene expression differences associated with ileal microbial community composition

We next applied MaAsLin ${ }^{19,20}$ in order to test specifically for associations between age-dependent CD host gene expression and specific microbial taxonomy factor $(n=229)$. We included in the analysis differentially expressed genes from selected representative pathways (Figs. 1 and 2) including DEFA5, GSTA1, and LCT that were downregulated and CSF2, CXCR1, IFNG, and MMP1 that were upregulated in older vs. younger $C D$. We also included clinical phenotype $(\mathrm{Ctl}, \mathrm{CD})$, endoscopic severity (ileal deep ulcers), clinical severity (PCDAl), age-of-diagnosis ( $\geq 10$ years vs. $<10$ years), gender, BMI, recent exposure to antibiotics, and NOD2, and ATG16L1 risk allele carriage. Overall, we identified 38 significant associations for clinical phenotype, and 5 significant associations with host gene expression (Fig. 5).

The bar graph (Fig. 5a) illustrates fold change differences between $C D$ and $\mathrm{Ctl}$ taking into account the clinical factors and host gene expression. As was previously reported by us ${ }^{19,20}$ and others, ${ }^{32} \mathrm{CD}$ phenotype showed a decrease in taxa from the Firmicutes phylum including Faecalibacterium prausnitzii, Lachnospiraceae, and Ruminococcaceae OTUs and an increase in taxa from the Proteobacteria and Fusobacteria phyla including Pasteurellaceae, Campylobacteraceae, Enterobacteriaceae, and Fusobacteriaceae organisms. Further, we determined a positive associations between Bacteroides abundance and a-defensin expression and a negative association between Acinetobacter and a-defensin expression. Finally, we noted a negative association between Lachnospiraceae abundance and IFNY expression and between Bacteroides fragilis and MMP1 expression. No significant associations were captured for CSF2, CXCR1, GSTA1, and LCT host gene expression. Altogether, these results demonstrated that while some of the age-dependent host gene expression dynamics were associated with specific microbial taxa, gene expression differences were largely not associated with systematic microbial shifts within $C D$ age-of-diagnosis.

\section{DISCUSSION}

Epidemiologic associations and clinical phenotype, as well as natural history, differ across CD age-of-diagnosis. ${ }^{1-4}$ Using the RISK cohort, we were able to show that while there were no significant differences in baseline clinical severity (PCDAI), early exposure to anti-TNFa, ${ }^{18}$ and PCDAl six month after diagnosis between older (A1b) and younger (A1a) CD, however, the prevalence of penetrating complications (B3) during follow up was significantly higher in older CD (Table 1). By using whole transcriptome mRNAseq analyses, we were able to identify 365 genes that were differentially expressed between older and younger $\mathrm{iCD}$, with older iCD showing an increased Th1-related IFNy profile associated with amplified inflammatory activation including an enriched innate myeloid and lymphoid stromal cell signature, and enhanced extracellular matrix and collagen signatures. Remarkably, this signature for enhanced immune activation occurred in the older iCD group in association with a specific reduction in epithelial Paneth cell a-defensin expression, and was not associated with systematic changes in the local mucosal microbial communities across $C D$ age-of-diagnosis (Summarizing cartoon, Fig. 6). We therefore suggest that age-dependent ileal host inflammatory intensification and depression of a-defensins genes is largely intrinsic to the mucosal dynamics and is not associated with a systematic local microbial community shift.

While a reduction in a-defensins was previously described in ileal $C D$, there was an ongoing controversy as to whether NOD2 and ATG16L1 genotype and/or the presence of inflammation is the primary drive of low a-defensin levels. ${ }^{16,33,34}$ We detected preservation of DEFA5/DEFA6 expression in the ileum of younger iCD patients similar to $\mathrm{Ctl}$ levels, and a significant reduced expression in older iCD patients. We specifically show that older age-of-diagnosis is associated with decreased expression of 
Paneth-cell associated $a$-defensins and that these age-related differences cannot be explained solely by either NOD2 or ATG16L1 genotype. We did not however identify similar decreased expression of TCF4, ${ }^{35}$ LRP6, $^{36}$ and TCF $^{37}$ (also known as TCF-1) which were previously linked to a-defensin expression in iCD. DNASE1 that was negatively associated with abnormal Paneth cell morphology in $\mathrm{CCD}^{29}$ showed a similar pattern of lower expression level in older $\mathrm{iCD}$ as the a-defensins and $R E G 3 G / A$ in contrast to stable increased expression of lysozyme ( $L Y Z)$ that was one of the differentially expressed core iCD genes ${ }^{19}$ but not included in the 365 genes (Suppl. Table 2). We did identify a negative association between IFNy and a-defensins expression, consistent with the previously reported bi-directional negative regulation of these genes. ${ }^{31}$ Th1-related IFN $\gamma$ was suggested to specifically repress adefensin gene expression by leading to Paneth cells extrusion and subsequent cell death. ${ }^{30}$

Early life gut microbial maturation and its implications in health and disease were previously characterized. ${ }^{38}$ By the end of the third year of life, the microbiome composition evolves toward an adult-like configuration. ${ }^{39}$ However, a specific understanding of the microbiome of older pediatric cohorts ( $>3$ years) and preadulthood ( $<18$ years) has been limited, ${ }^{40,41}$ whereby the largest cohort included 62 stool samples from residents of the United States. $^{39}$ Our cohort ${ }^{19,20}$ includes $178 \mathrm{Ctl}$ with ileal mucosal microbial composition. We were able to show that dynamic microbial changes occur in the ileum of older $\mathrm{Ctl}$ cases in comparison to younger $\mathrm{Ctl}$ cases, with no similar significant association detected in in older vs. younger $C D$ patients. Collectively these data show that the $C D$ dysbiosis characterized by increased abundance of pro-inflammatory taxa including taxa from Enterobacteriaceae, Pasteurellaceae, Veillonellaceae, and Fusobacteriaceae families, and decreased abundance of antiinflammatory taxa from the Erysipelotrichaceae, Ruminococcaceae and Lachnospiraceae families was largely independent of CD ageof-diagnosis.

To test for associations between the microbiota, and agedependent ileal gene expression, we specifically performed multivariate analysis that included $\mathrm{Ctl}$ versus $\mathrm{CD}$ clinical phenotype, age-of-diagnosis, and host genes that were differentially expressed in older vs. younger CD. Indeed, we were able to identify a significant association with expression of specific Th1 and tissue remodeling genes (IFNY and MMP1), which were upregulated in older CD, and Lachnospiraceae and Bacteroides fragilis OTU abundance respectively. We also identified a significant positive association between Bacteroides abundance and negative association between Acinetobacter, and the antimicrobial gene DEFA5. Importantly, DEFA5-transgenic mice also showed a significant increase in taxa from the Bacteroidetes phylum. ${ }^{15}$ These data demonstrate that while some of the mucosal gene expression differences were associated with the abundance of specific microbial taxa, much of the robust agedependent mucosal immune gene signature maturation observed is likely driven by host mucosal factors. However, with further defining of the changes in intestinal microbiota, specific strains may be found to play important in mucosal gene expression. For instance, a Faecalibacterium prausnitzii out was found to be decreased but there are also other $F$. prausnitzii OTUs that are increased in $C D$ suggesting potential strain-specificity. ${ }^{42}$

Our study has several strengths, but also some limitations. Although it is reasonable to assume that puberty influences the observed gut maturation, we lacked consistent Tanner Stage information to specifically address this hypothesis. However, the clinical practice approach, namely the Paris classification, is specifically based on age-of-diagnosis and not on Tanner Stage. Future studies will need to test whether Tanner Stage and puberty-related hormones can further define primary pathways driving mucosal gene expression maturation in association with disease onset at specific ages. Because we require OTUs to occur at a relative abundance of at least $0.01 \%$ across all samples, it remains possible that a rare but infrequent immunomodulatory organism might explain the differences in the younger and older group. Moreover, we lacked power to test for differences in mucosal gene expression between sub-sets of younger and older patients stratified by measures of microbial diversity. Additionally, we used whole biopsies, composed of a mixture of cellular components rather than single cell transcriptomics. However, in order to capture the overall pathogenic process, and as a potential future diagnostic/prognostic tool, there are also substantial advantages in using whole mucosal biopsies, which are the basis for diagnosis and follow up in the clinical setting. Another limitation is the inability to replicate this results in an independent large treatment naïve human cohort not affected by treatment regimens. RISK is the largest treatment naïve inception cohort, involving 28 sites in the US, and other such large human cohorts are not yet available. However, there are several murine and mechanistic studies supporting our findings. Age-specific response to enteric Salmonella infection, with developmentally regulated intestinal expression of IFN- $\gamma$ and its target genes was already noted. ${ }^{13}$ Paneth cells degranulation does not directly occur upon stimulation with microbial antigens or bacteria, but IFN- $\gamma$ induces rapid and complete loss of Paneth cells granules, coupled with induction of apoptosis, luminal extrusion, and death of Paneth cells. ${ }^{30}$ Finally, similar to our findings, Firmicutes was significantly lower and Bacteroidetes was significantly higher in the DEFA5 transgenic $(+/+)$ mice than in controls. ${ }^{15}$

In summary, our data identify important clinical and biologic differences between older and younger pediatric $C D$. We would suggest that pediatric $A 1 b C D$ phenotype and mucosal gene expression signature, is likely very similar to adult (A2) $C D, 16,43,44$ and different from the younger pediatric age group. Interestingly, in mice and humans the initial formation of Peyer's patches occurs before birth in a relative sterile environment and hence is likely independent of microbiota. ${ }^{45}$ Consistent with this observation, our data suggest that much of the age-dependent differentially expressed gene expression signature in pediatric $C D$ ( $\geq 10$ years) is intrinsic to the host ileal mucosa, and not primarily associated with systematic shifts in the local microbial community. These specific host and microbial factors may offer the potential to tailor future age-based therapy.

Transcript profiling

The ileal RNASeq data has been placed in the GEO repository with the following accession number: GSE101794.

\section{ACKNOWLEDGEMENTS}

This work was supported by the Crohn's and Colitis Foundation, the Gene and Protein Expression and Bioinformatics cores of the National Institutes of Health (NIH)supported Cincinnati Children's Hospital Research Foundation Digestive Health Center (1P30DK078392-01), NIH grant U54 DE023798 and HMP2 (R.J.X., and C.H), the Leona M. and Harry B. Helmsley Charitable Trust (R.J.X., and C.H), the European Crohn's and Colitis Organization (ECCO, Y.H), the Israel Science Foundation (grant No 908/15), the I-CORE program (Y.H), and European Research Council starting grant (grant No 758313, Y.H). We thank the Crohn's and Colitis Foundation RISK study publication committee for critical review of this manuscript.

\section{ADDITIONAL INFORMATION}

The online version of this article (https://doi.org/10.1038/s41385-018-0114-4) contains supplementary material, which is available to authorized users.

Competing interests: The authors declare no competing interests. 


\section{REFERENCES}

1. Ruel, J., Ruane, D., Mehandru, S., Gower-Rousseau, C. \& Colombel, J. F. IBD across the age spectrum-is it the same disease? Nature reviews. Gastroenterol. \& Hepatol. 11, 88-98 (2014).

2. Siegel, C. A. et al. Real-time tool to display the predicted disease course and treatment response for children with Crohn's disease. Inflamm. Bowel Dis. 17, 30-38 (2011).

3. Gower-Rousseau, C. et al. Epidemiology of inflammatory bowel diseases: new insights from a French population-based registry (EPIMAD). Dig. Liver Dis. : Off. J. Ital. Soc. Gastroenterol. Ital. Assoc. Study Liver 45, 89-94 (2013).

4. Heyman, M. B. et al. Children with early-onset inflammatory bowel disease (IBD): analysis of a pediatric IBD consortium registry. J. Pediatr. 146, 35-40 (2005).

5. Meinzer, U. et al. lleal involvement is age dependent in pediatric Crohn's disease. Inflamm. Bowel Dis. 11, 639-644 (2005).

6. Levine, A. et al. Pediatric modification of the Montreal classification for inflammatory bowel disease: the Paris classification. Inflamm. Bowel Dis. 17, 1314-1321 (2011).

7. Uhlig, H. H. et al. The diagnostic approach to monogenic very early onset inflammatory bowel disease. Gastroenterology 147, 990-1007 e1003 (2014).

8. Muise, A. M., Snapper, S. B. \& Kugathasan, S. The age of gene discovery in very early onset inflammatory bowel disease. Gastroenterology 143, 285-288 (2012).

9. de Bie, C. I. et al. Diagnostic workup of paediatric patients with inflammatory bowel disease in Europe: results of a 5-year audit of the EUROKIDS registry. J. Pediatr. Gastroenterol. Nutr. 54, 374-380 (2012).

10. Cornes, J. S. Number, size, and distribution of Peyer's patches in the human small intestine: Part I The development of Peyer's patches. Gut 6, 225-229 (1965).

11. Van Kruiningen, H. J., Ganley, L. M. \& Freda, B. J. The role of Peyer's patches in the age-related incidence of Crohn's disease. J. Clin. Gastroenterol. 25, 470-475 (1997).

12. Jung, C., Hugot, J. P. \& Barreau, F. Peyer's Patches: the immune sensors of the intestine. Int. J. Inflam. 2010, 823710 (2010).

13. Rhee, S. J., Walker, W. A. \& Cherayil, B. J. Developmentally regulated intestinal expression of IFN-gamma and its target genes and the age-specific response to enteric Salmonella infection. J. Immunol. 175, 1127-1136 (2005).

14. Salvati, V. M. et al. Enhanced expression of interferon regulatory factor-1 in the mucosa of children with celiac disease. Pediatr. Res. 54, 312-318 (2003).

15. Salzman, N. H. et al. Enteric defensins are essential regulators of intestinal microbial ecology. Nat. Immunol. 11, 76-83 (2010).

16. Wehkamp, J. et al. Reduced Paneth cell alpha-defensins in ileal Crohn's disease. Proc. Natl Acad. Sci. USA. 102, 18129-18134 (2005).

17. Ramasundara, M., Leach, S. T., Lemberg, D. A. \& Day, A. S. Defensins and inflammation: the role of defensins in inflammatory bowel disease. J. Gastroenterol. Hepatol. 24, 202-208 (2009).

18. Kugathasan, S. et al. Prediction of complicated disease course for children newly diagnosed with Crohn's disease: a multicentre inception cohort study. Lancet, https://doi.org/10.1016/S0140-6736(17)30317-3 (2017).

19. Haberman, Y. et al. Pediatric Crohn disease patients exhibit specific ileal transcriptome and microbiome signature. J. Clin. Invest. 124, 3617-3633 (2014).

20. Gevers, D. et al. The treatment-naive microbiome in new-onset Crohn's disease. Cell. Host. Microbe 15, 382-392 (2014).

21. Dubinsky, M. C. et al. Increased immune reactivity predicts aggressive complicating Crohn's disease in children. Clin. Gastroenterol. Hepatol. 6, 1105-1111 (2008).

22. Han, X. et al. Granulocyte-macrophage colony-stimulating factor autoantibodies in murine ileitis and progressive ileal Crohn's disease. Gastroenterology 136, 1261-1271 (2009). e1261-1263.

23. Bray, N. L., Pimentel, H., Melsted, P. \& Pachter, L. Near-optimal probabilistic RNAseq quantification. Nat. Biotechnol. 34, 525-527 (2016).

24. Chen, J., Bardes, E. E., Aronow, B. J. \& Jegga, A. G. ToppGene Suite for gene list enrichment analysis and candidate gene prioritization. Nucleic Acids Res. 37, W305-W311 (2009).

25. Kaimal, V., Bardes, E. E., Tabar, S. C., Jegga, A. G. \& Aronow, B. J. ToppCluster: a multiple gene list feature analyzer for comparative enrichment clustering and network-based dissection of biological systems. Nucleic Acids Res. 38, W96-W102 (2010).

26. Saito, R. et al. A travel guide to Cytoscape plugins. Nat. Methods 9, 1069-1076 (2012).

27. Caporaso, J. G. et al. Ultra-high-throughput microbial community analysis on the Illumina HiSeq and MiSeq platforms. Isme. J. 6, 1621-1624 (2012).

28. McDonald, D. et al. An improved Greengenes taxonomy with explicit ranks for ecological and evolutionary analyses of bacteria and archaea. Isme. J. 6, 610-618 (2012).

29. Vandussen, K. L. et al. Genetic variants synthesize to produce paneth cell phenotypes that define subtypes of Crohn's disease. Gastroenterology 146, 200-209 (2014).

30. Farin, H. F. et al. Paneth cell extrusion and release of antimicrobial products is directly controlled by immune cell-derived IFN-gamma. J. Exp. Med. 211, 1393-1405 (2014)

31. Biswas, A. et al. Induction and rescue of Nod2-dependent Th1-driven granulomatous inflammation of the ileum. Proc. Natl Acad. Sci. USA. 107, 14739-14744 (2010).

32. Frank, D. N. et al. Molecular-phylogenetic characterization of microbial community imbalances in human inflammatory bowel diseases. Proc. Natl Acad. Sci. USA. 104, 13780-13785 (2007).

33. Simms, L. A. et al. Reduced alpha-defensin expression is associated with inflammation and not NOD2 mutation status in ileal Crohn's disease. Gut 57, 903-910 (2008).

34. Shanahan, M. T. et al. Mouse Paneth cell antimicrobial function is independent of Nod2. Gut, https://doi.org/10.1136/gutjnl-2012-304190 (2013).

35. Perminow, G. et al. Defective paneth cell-mediated host defense in pediatric ileal Crohn's disease. Am. J. Gastroenterol. 105, 452-459 (2010).

36. Koslowski, M. J. et al. Association of a functional variant in the Wnt co-receptor LRP6 with early onset ileal Crohn's disease. PLoS. Genet. 8, e1002523 (2012).

37. Beisner, J. et al. TCF-1 mediated Wnt Signaling regulates Paneth cell innate immune defense effectors HD-5 and -6: implications for Crohn's disease. American journal of physiology. Gastrointestinal and liver physiology, https://doi.org/ 10.1152/ajpgi.00347.2013 (2014)

38. Yatsunenko, T. et al. Human gut microbiome viewed across age and geography. Nature 486, 222-227 (2012).

39. Gordon, J. I., Dewey, K. G., Mills, D. A. \& Medzhitov, R. M. The human gut microbiota and undernutrition. Sci. Transl. Med. 4, 137ps112 (2012).

40. Harris, R. A. et al. Colonic mucosal epigenome and microbiome development in children and adolescents. J. Immunol. Res. 2016, 9170162 (2016).

41. Hollister, E. B. et al. Structure and function of the healthy pre-adolescent pediatric gut microbiome. Microbiome 3, 36 (2015).

42. Assa, A. et al. Mucosa-associated ileal microbiota in new-onset pediatric Crohn's Disease. Inflamm. Bowel Dis. 22, 1533-1539 (2016).

43. Noble, C. L. et al. Characterization of intestinal gene expression profiles in Crohn's disease by genome-wide microarray analysis. Inflamm. Bowel Dis. 16, 1717-1728 (2010).

44. Arijs, I. et al. Predictive value of epithelial gene expression profiles for response to infliximab in Crohn's disease. Inflamm. Bowel Dis. 16, 2090-2098 (2010).

45. Renz, H., Brandtzaeg, P. \& Hornef, M. The impact of perinatal immune development on mucosal homeostasis and chronic inflammation. Nat. Rev. Immunol. 12, 9-23 (2012). 